\title{
Resolving oscillation degeneracies with a single neutrino polarity
}

\section{Davide Meloni}

Dipartimento di Fisica, Universitá di Roma Tre and INFN, Sez. di Roma Tre, Roma, Italy. meloniefis.uniroma3.it

\section{Olga Mena}

Institut de Ciéncies de l'Espai, Campus de Ciéncies, Bellaterra, Spain.

mena@aliga.ieec.uab.es

\section{Christopher Orme* and Silvia Pascoli}

IPPP, Department of Physics, Durham University, Durham DH1 3LE, United Kingdom.

c.d.orme@durham.ac.uk and silvia.pascoli@durham.ac.uk

\section{Sergio Palomares-Ruiz}

CFTP, Departamento de Física, Instituto Superior Técnico, Lisboa, Portugal.

sergio.palomares.ruiz@ist.utl.pt

\begin{abstract}
Within the future programme of neutrino oscillation experiments that is currently under discussion, long baseline experiments will play a crucial role in providing information on the value of $\theta_{13}$, the type of neutrino mass ordering and on the value of the CP-violating phase $\delta$, which enters in 3-neutrino oscillations. Here, we consider a beta-beam setup with an intermediate Lorentz factor $\gamma=450$ and a baseline of $1050 \mathrm{~km}$. This could be achieved in Europe with a beta-beam sourced at CERN to a detector located at the Boulby mine in the United Kingdom. We consider a neutrino run alone and show that, by exploiting the oscillatory pattern of the signal, a very good sensitivity to $\mathrm{CP}$-violation and the type of hierarchy can be reached. We analyse the physics potential of this setup in detail studying two different exposures $\left(1 \times 10^{21}\right.$ and $5 \times 10^{21}$ ions-ktonyears). In both cases, we find that the type of neutrino mass hierarchy could be determined at $99 \%$ $\mathrm{CL}$, for all values of $\delta$, for $\sin ^{2} 2 \theta_{13}>0.03$. In the high-exposure scenario, we find that the value of the CP-violating phase $\delta$ could be measured with a $99 \%$ CL error of $\sim 20^{\circ}$ if $\sin ^{2} 2 \theta_{13}>10^{-3}$, with some sensitivity down to values of $\sin ^{2} 2 \theta_{13} \simeq 10^{-4}$.
\end{abstract}

10th International Workshop on Neutrino Factories, Super beams and Beta beams June 30 - July 52008

Valencia, Spain

\footnotetext{
*Speaker.
} 


\section{Introduction}

In this poster, we discuss how neutrino oscillation degeneracies could be overcome through the use of a single neutrino polarity, taking an intermediate boost beta-beam as a case study. We present a detailed numerical simulation confirming the analytical study presented in [1].

\section{Simulation and results}

We consider boosted ${ }^{18} \mathrm{Ne}$ ions directed along the CERN-Boulby baseline $(1050 \mathrm{~km})$ with a conservative Lorentz factor (for an upgraded SPS) of $\gamma=450$. We first consider an exposure corresponding to $10^{21}$ ions-kton-years. This could be obtained, for example, assuming $2 \times 10^{18}$ useful ion decays per year and a 50 kton detector with $100 \%$ efficiency located at Boulby with 10 years of data taking. We also upgrade the first scenario by a factor of five in statistics. We assume a $200 \mathrm{MeV}$ bin width and an energy detection threshold of $400 \mathrm{MeV}$. The muon-neutrino appearance signal has been binned in eleven bins with a bin width of $200 \mathrm{MeV}$ in the $[0.4,2.0]$ $\mathrm{GeV}$ energy range, plus a unique, last bin, filled with the neutrino events from $2.0 \mathrm{GeV}$ up to the end point of the spectrum at $3.06 \mathrm{GeV}$. We have taken overall systematics of $2 \%$, an intrinsic beam background of $0.1 \%$, and used atmospheric backgrounds of 0.03 per kton-yr assuming a duty factor of $10^{-3}$.

We present in Fig. 1 the 90\%, 95\% and 99\% CL contours for a fit to the simulated data from the beta-beam experiment described in the previously. The simulations are for the normal mass hierarchy and $\theta_{23}$ in the first octant $\left(\sin ^{2} \theta_{23}=0.41\right.$ which corresponds to $\theta_{23}=40^{\circ}$ ). The statistics considered corresponds to the optimistic high-statistics scenario. The analysis depicted in Fig. 1 includes the study of the discrete degeneracies.
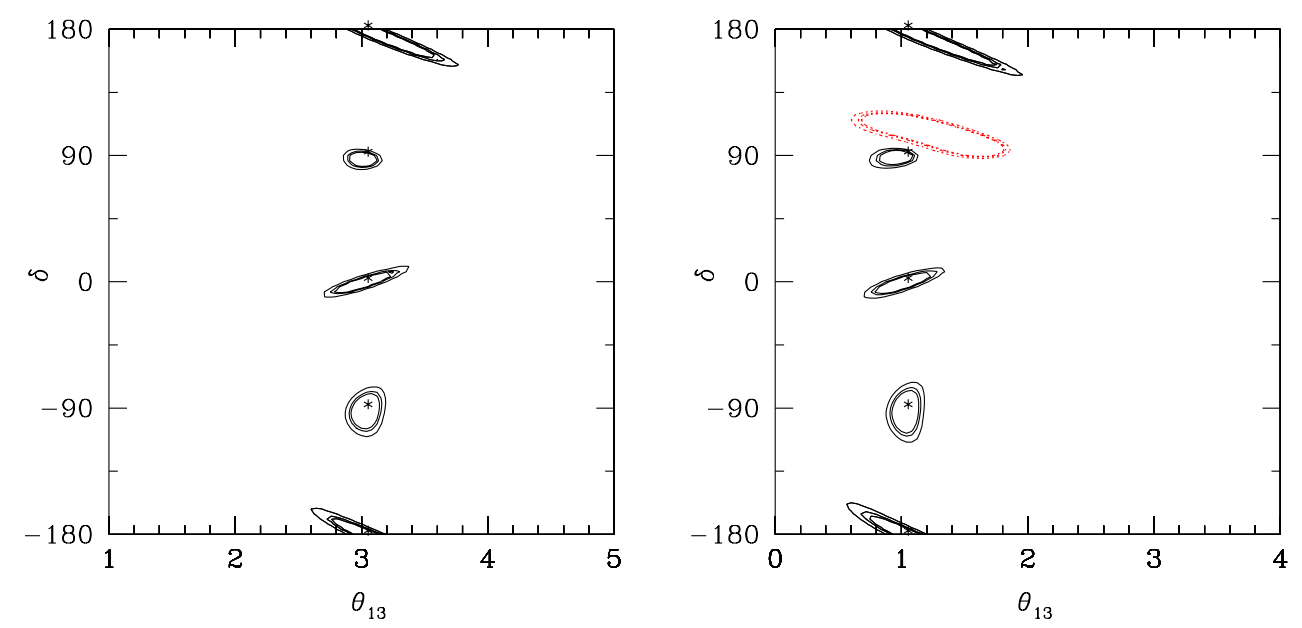

Figure 1: For an exposure of $5 \times 10^{21}$ ions-kton-years, 90\%, 95\% and 99\% (for 2 d.o.f) CL contours resulting from the fits if the true values Nature has chosen are $\theta_{13}=3^{\circ}$ (left panel) or $\theta_{13}=1^{\circ}$ (right panel), and $\delta=0^{\circ}, 90^{\circ},-90^{\circ}$ or $180^{\circ}$. Dashed-red contours represent the hierarchy-clone solution.

Figs. 2 summarises, for the low- and high-statistics scenarios, the physics reach of the betabeam experiment considered here. The analysis takes into account the impact of both the intrinsic 

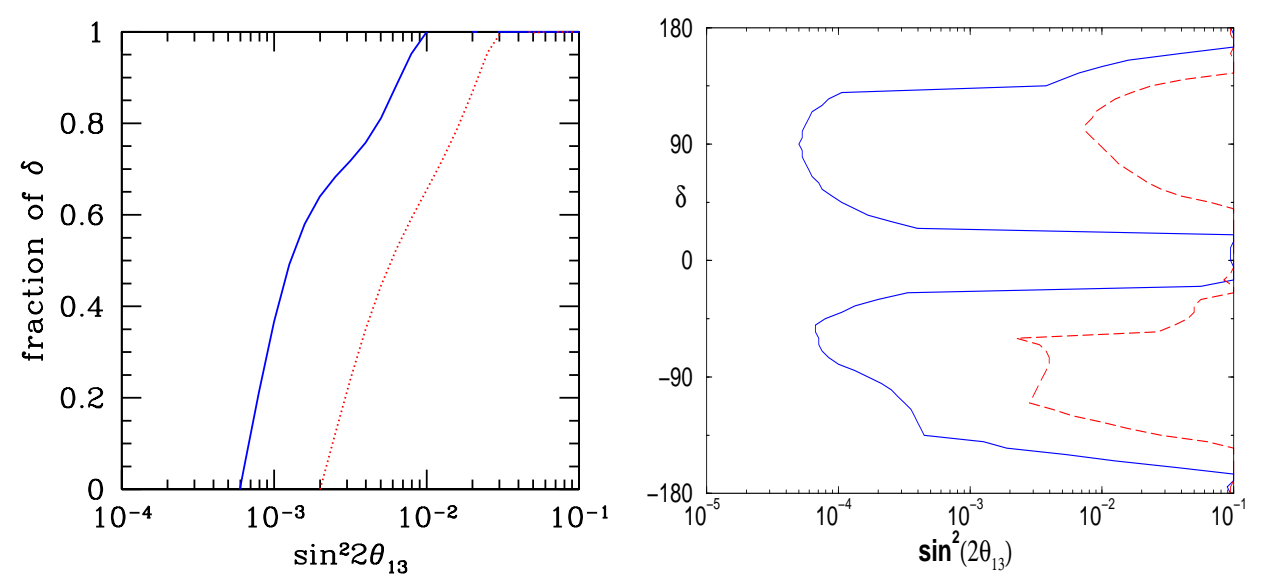

Figure 2: Left: $99 \%$ CL hierarchy resolution (2 d.o.f) and right: :Discovery at $99 \%$ CL of CP violation (2 d.o.f). The solid blue curve depicts the results assuming the statistics quoted before is improved by a factor of five.

and discrete degeneracies. Fig. 2 (left) shows the region in the ( $\sin ^{2} 2 \theta_{13}$, "fraction of $\delta$ ") plane for which the mass hierarchy can be resolved at the 99\% CL ( 2 d.o.f). Fig. 2 (right) shows the region in the $\left(\sin ^{2} 2 \theta_{13}, \delta\right)$ plane for which a given (non-zero) value of the CP-violating phase $\delta$ can be distinguished at the $99 \% \mathrm{CL}$ ( 2 d.o.f.) from the $\mathrm{CP}$-conserving case, i.e., $\delta=0, \pm 180^{\circ}$. The results are given for both the low- and high-statistics scenarios.

\section{Summary}

In the present article, we have reviewed the physics reach of a beta-beam with intermediate $\gamma$ and long baseline. We have considered a neutrino beam sourced by ${ }^{18} \mathrm{Ne}$ decays with $\gamma=450$ and a baseline of $1050 \mathrm{~km}$ corresponding to the CERN-Boulby mine distance. We have shown that, by exploiting the oscillatory behaviour of the signal, it is possible to fully resolve such degeneracies in a large part of the allowed parameter space.

\section{Acknowledgments}

OM was supported by the European Programme "The Quest for Unification" under contract MRTN-CT-2004-503369. CO acknowledges the support of a STFC studentship. SPR is partially supported by the Spanish Grant FPA2005-01678 of the MCT. SP acknowledges the support of CARE, contract number RII3-CT-2003-506395.

\section{References}

[1] D. Meloni, O. Mena, C. Orme, S. Palomares-Ruiz and S. Pascoli, JHEP 0807 (2008) 115 [arXiv:0802.0255 [hep-ph]]. 